\title{
Human Face-Selective Cortex Does Not Distinguish between Members of a Racial Outgroup
}

\author{
일 Reggev, ${ }^{1,2,3}$ Kirstan Brodie, ${ }^{1}$-Mina Cikara, ${ }^{1}$ and Jason P. Mitchell ${ }^{1}$
}

https://doi.org/10.1523/ENEURO.0431-19.2020

${ }^{1}$ Department of Psychology, Harvard University, Cambridge, MA 02138, 2Department of Psychology, Ben Gurion University of the Negev, Be'er-Sheva 84105, Israel, and 'Zlotowski Center for Neuroscience, Ben Gurion University of the Negev, Be'er-Sheva 84105, Israel

\begin{abstract}
People often fail to individuate members of social outgroups, a phenomenon known as the outgroup homogeneity effect. Here, we used functional magnetic resonance imaging (fMRI) repetition suppression to investigate the neural representation underlying this effect. In a preregistered study, White human perceivers $(N=29)$ responded to pairs of faces depicting White or Black targets. In each pair, the second face depicted either the same target as the first face, a different target from the same race, or a scrambled face outline. We localized face-selective neural regions via an independent task, and demonstrated that neural activity in the fusiform face area (FFA) distinguished different faces only when targets belonged to the perceivers' racial ingroup (White). By contrast, face-selective cortex did not discriminate between other-race individuals. Moreover, across two studies (total $N=67$ ) perceivers were slower to discriminate between different outgroup members and remembered them to a lesser extent. Together, these results suggest that the outgroup homogeneity effect arises when early-to-mid-level visual processing results in an erroneous overlap of representations of outgroup members.
\end{abstract}

Key words: cross-race; faces; FFA; fMRI; outgroup homogeneity; repetition suppression

\section{Significance Statement}

Researchers have repeatedly demonstrated that perceivers struggle to distinguish between different members of a racial outgroup. Here, we show in a preregistered study that this failure arises when areas of the human brain that specifically process facial identity, most notably, the so-called "fusiform face area" (FFA), fail to detect differences between identities of members of a racial outgroup. When White perceivers viewed photographs of two different Black men, the face area of their brains responded as if the two photographs portrayed the same person. This effect was constrained to outgroup faces; the face area successfully distinguished faces of two different White individuals. Our results highlight the failure of basic representational mechanisms in processing individuals from other social groups.

\section{Introduction}

The outgroup homogeneity effect (sometimes called the cross-race effect) describes the difficulty people often experience when trying to identify members of a racial outgroup. Although race is not a valid biological taxonomy, individuals in our society define separate human races by sociocultural experiences and use this social

Received October 16, 2019; accepted April 9, 2020; First published May 18, 2020.

The authors declare no competing financial interests. taxonomy as a basis for numerous social and cognitive processes (Wagner et al., 2017). For example, White perceivers typically remember faces of White targets better than faces of Black targets, and are more likely to say that a new, unfamiliar Black face is the same as one they have seen previously (Malpass and Kravitz, 1969; Meissner and Brigham, 2001). Perceivers also identify own-race faces

Author contributions: N.R., K.B., M.C., and J.P.M. designed research; N.R. performed research; N.R. analyzed data; N.R., K.B., M.C., and J.P.M. wrote the paper. 
faster and more accurately than cross-race faces (Marcon et al., 2010). This pattern of (mis)identification has demonstrable societal consequences. In an analysis of American police proceedings, White witnesses correctly identified $60 \%$ of perpetrators when a line-up comprised other White individuals but identified only $45 \%$ of Black perpetrators; more than half the time, unrelated (i.e., innocent) Black individuals were identified as perpetrators (Behrman and Davey, 2001).

Most theories of the outgroup homogeneity effect suggest that perceivers create detailed, individuated representations of ingroup members, but view outgroup members as interchangeable instances of a category (Papesh and Goldinger, 2010; Hugenberg et al., 2013; Correll et al., 2017). Consequently, scholars hypothesize that this differential representation underlies perceivers' improved memory for members of their ingroup, as well as their heightened judgments of diversity and distinctiveness for ingroup targets (Park and Rothbart, 1982; Judd et al., 2005; Boldry et al., 2007). However, research to date has measured representations of racial ingroup and outgroup members mainly as a function of behavioral responses to ingroup and outgroup targets (e.g., reaction time differences between different targets; Papesh and Goldinger, 2010). To the best of our knowledge, no study has directly measured the target-specific representations of members of different groups, which we hypothesize underlie the previously mentioned behavioral results.

To bridge this gap, we conducted a functional magnetic resonance imaging (fMRI) study that made use of the phenomenon of repetition suppression, whereby neural responses to a repeated stimulus are reduced (or suppressed) relative to a sequence of two different stimuli (Gotts et al., 2012). For example, regions of the human brain that respond robustly to faces, such as the fusiform gyrus, will decrease their activity when participants view the same face repeatedly. By contrast, activation in this region will return to typically high levels of activation when a new face is presented (Gilaie-Dotan et al., 2010). Accordingly, this "release from suppression" can be used to measure the degree to which perceivers detect that they have viewed the faces of two different individuals. Thus, our approach extends previous neuroimaging studies that have, for the most part, characterized differences in mean levels of neural activity between social groups (Golby et al., 2001; Van Bavel et al., 2008; Mathur et al., 2012). Whereas these traditional univariate approaches can offer insights into the localization of neural activity

This work was partially supported by the Israeli Science Foundation Grant 79/18 (to N.R.) and was conducted at the Harvard University Center for Brain Science, which is supported by the National Institutes of Health Shared Instrumentation Grant Program S100D020039.

Acknowledgements: We thank J. Ontko for insights and assistance in data collection.

Correspondence should be addressed to Niv Reggev at reggevn@bgu.ac.il. https://doi.org/10.1523/ENEURO.0431-19.2020 Copyright (C) 2020 Reggev et al.

This is an open-access article distributed under the terms of the Creative Commons Attribution 4.0 International license, which permits unrestricted use, distribution and reproduction in any medium provided that the original work is properly attributed. differences in response to social groups, the release from suppression effect, by contrast, characterizes the uniqueness (or similarity) of representations of distinct Black and distinct White faces.

Here, we make use of the release-from-suppression logic to examine the representational basis of the outgroup homogeneity effect. Researchers have shown that the fusiform face area (FFA) shows repetition suppression even when participants see a single individual from different angles (Pourtois et al., 2005) or from different distances (Grill-Spector et al., 1999), suggesting that the FFA is sensitive to the identity of an individual rather than the similarity of their perceptual features. We capitalized on these characteristics to examine whether White perceivers will likewise represent two different Black individuals as more similar to each other than two White individuals. Specifically, we hypothesized that if perceivers individuate ingroup and outgroup faces equally, then we should observe similar release from suppression for two different face identities regardless of their race. However, to the extent that White perceivers are worse at "detecting a difference" between faces of different Black targets, then the FFA should show more suppression when a Black face is followed by a new, different Black face, despite the second face being different from the first (for related approaches, see Vizioli et al., 2010; Hughes et al., 2019). Importantly, only identity-sensitive regions such as the FFA should show the differential suppression for Black faces; face-specific identity-insensitive regions [e.g., occipital face area (OFA)] should show equivalent suppression for different Black and White faces.

To test these predictions, we conducted a behavioral experiment and a preregistered fMRI study using a repetition suppression paradigm. In each experiment, White participants sequentially viewed pairs of faces that varied in race (Black, White) and gender (woman, man). We matched face categories in perceptual and structural properties (see Materials and Methods). For each pair, participants indicated whether the faces were of the same or different individuals. In some trials, the two faces were identical; in an equal number of trials, the two faces depicted different individuals of the same race and gender. In addition, in one-third of trials in the fMRI experiment, the second face was replaced by a scrambled faceshaped patch; this condition allowed us to establish a difference in baseline neural processing of Black and White faces, and to directly replicate earlier studies (Golby et al., 2001; Van Bavel et al., 2011; Fig. 1). Our behavioral pilot studies indicated that participants demonstrated a reliable race effect only for male targets. Therefore, our confirmatory analyses focused on male targets; we report the results of the exploratory analyses for female targets in the supporting information. Notably, most previous studies of the other-race homogeneity effect have included only male targets; here, we provide an initial attempt to address this empirical lacuna.

\section{Materials and Methods}

Human participants were recruited from the local community using the Harvard Department of Psychology 
A

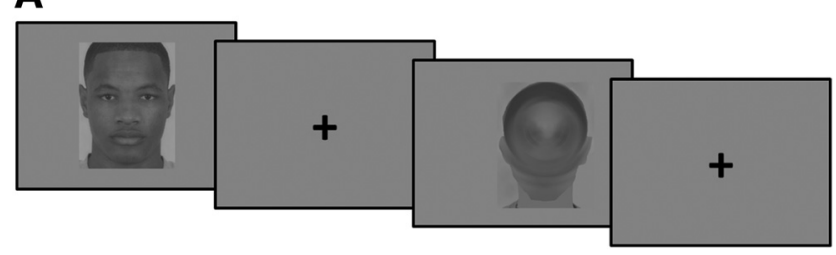

B

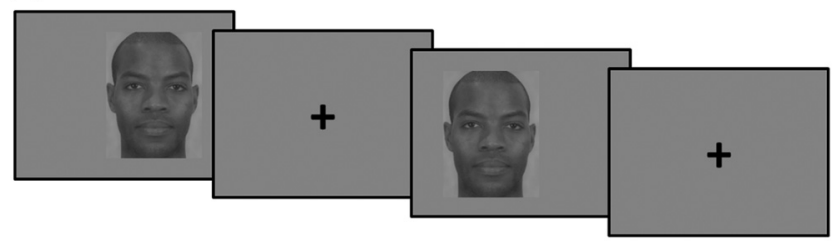

C

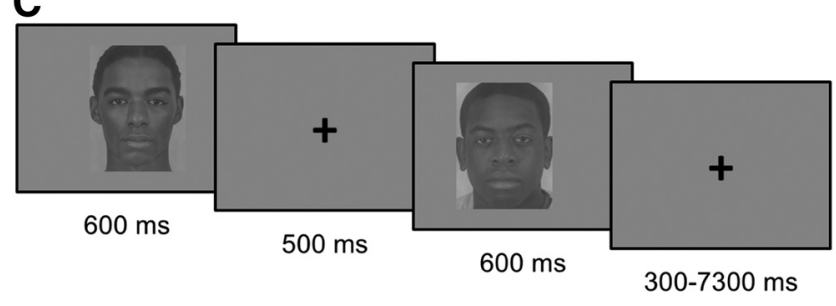

Same/Diff?

Figure 1. Task design. In the fMRI scanner, participants viewed face pairs from different races (Black, White) and genders (women, men) and decided if the two faces belonged to the same individual. Participants saw each face for $600 \mathrm{~ms}$ and responded when the second face was on screen. Faces were presented with a random horizontal jitter to prevent low-level suppression effects. The design included three face pair conditions for each combination of race and gender. Participants saw $(\boldsymbol{A})$ a face followed by a scrambled visual patch in the shape of a face in the single condition (i.e., baseline trials; we included this condition only in experiment 2$),(\boldsymbol{B})$ a face followed by the same face in the repeated-face condition, and $(\boldsymbol{C})$ a face followed by a different face (matched in gender and race) in the different-face condition. We report the behavioral results in Figure 2 (male targets) and Extended Data Figure 1-1 (female targets).

Study Pool website. All participants provided their informed consent in a manner approved by the Committee on the Use of Human Subjects in Research at Harvard University.

\section{Experiment 1}

Thirty-eight self-identified White participants completed the experiment (22 female, 16 male; mean age: 21.00 , SD: 2.71, range: 16-29). We excluded one additional participant who failed to respond on time to $>20 \%$ of the trials in the face identification task. We collected the data on a rolling basis (multiple slots per day) with a target sample size of 32 participants. We identified this sample size to be sufficient to achieve a power of 0.8 to detect a hypothesized meaningful effect size estimate (Cohen's $d=0.3$, approximately equivalent to $\eta_{\mathrm{p}}^{2}=0.03$; Cohen, 1988, pp 276-281) for the interaction contrast of race (Black/White) by condition (repeated/different). Participants completed the experiment for course credit or financial compensation (US $\$ 10)$.

\section{Experiment 2}

Based on an identical power calculation, we aimed to collect analyzable data from 32 participants. To achieve this goal, we collected data from 38 self-identified White participants. In line with our preregistered exclusion criteria, we excluded six participants before data inspection due to lack of response to $>20 \%$ of the trials in the main task. In the main text, we report the results from 29 participants (15 female, 13 male, one non-binary; mean age: 21.97, SD: 3.02, range: 18-28) because we excluded three additional participants whose reaction time or accuracy data were $>2$ SDs beyond the sample mean (we report analysis with the full sample in the supporting information-the results do not change). All 32 participants provided adequate data in terms of signal quality, as measured by a slice signal-to-noise ratio higher than 150 and by having no more than three discrete movements larger than $0.5 \mathrm{~mm}$. All participants were healthy, right-handed, native English speakers with normal or corrected-to-normal vision, and no history of neurologic or psychiatric conditions. Participants completed the experiment for course credit or financial compensation (US\$50).

\section{Materials}

We obtained the face images for this study from the Chicago Face Database (CFD; Ma et al., 2015). We excluded faces that were identified as belonging to their respective racial group by fewer than $60 \%$ of the CFD independent raters, and faces that were identified as belonging to their racial group by between $60 \%$ and $75 \%$ of CFD raters were reviewed by two additional independent raters (N. R. and K. B.). Of these faces, 12 were excluded for having atypical features for their respective racial group, determined by interrater agreement between the additional raters. Lastly, all faces that were identified by $75 \%$ or more of CFD raters as belonging to their respective racial group were individually reviewed. Of these faces, two were excluded due to unique/distinctive facial features such as scars, and three were excluded due to noticeable artifacts in the image quality. This review process resulted in a final total of 94 Black female faces, 76 Black male faces, 77 White female faces, and 81 White male faces. This allowed us to select a total of 76 unique faces from each category for each participant (we used only 72 faces in experiment 1 as it required fewer stimuli; see task description below). We converted all images to greyscale, matched them on luminance (separately for foreground and background) using the SHINE toolbox (Willenbockel et al., 2010) for MATLAB (MathWorks), cropped them to 1246 by 946 pixels, matched them on spatial frequency using the SHINE toolbox, and finally, resized all photographs to 199 by 262 pixels. All face stimuli were presented in a rectangular box that included hair outline (Fig. 1).

To confirm that differences among the different categories were not confounded with image similarity, we measured the similarity between images by calculating the Structural Similarity Index (SSIM; Wang et al., 2004). This measure was computed after the experiment was completed. We computed the pairwise SSIM between all 
images in each category and averaged the SSIM score per image. This resulted in a vector of SSIM scores per category. Black and White men did not significantly differ in their average similarity (0.7169 vs 0.7148 , respectively; $\left.t_{(155)}=0.75, p=0.46\right)$. Black women, however, were less similar to each other compared with White women ( 0.7012 vs 0.7146 , respectively; $\left.t_{(155)}=5.31, p<0.001\right)$. This difference limits the potential interpretation of our exploratory analyses for female target faces (see Extended Data Fig. 3-5 and discussion below).

\section{Procedure}

We presented all tasks and stimuli via PsychoPy v1.84.2 (Peirce, 2007) running under Mac OS X 10.7 (experiment 1) or Windows 7 (experiment 2).

\section{Face repetition suppression task}

During the main task, we presented face images to participants in same-race, same-gender pairs. Experiment 1 included two conditions. In the repeated-face condition, the second face was identical to the first face. In the different-face condition, the second face was different from the first face. Experiment 2 included both the repeatedface condition and the different-face condition, as well as an additional single-face condition, where a single face was followed by a scrambled visual patch in the shape of a face; these pairs were used to establish a baseline. In experiment 1 , participants saw 24 face pairs per condition for each race (12 pairs for each gender). Experiment 2 included 38 face pairs per condition for each race (19 for each gender).

Each trial in the face task began with a face presented for $600 \mathrm{~ms}$, followed by a fixation crosshair presented in the center of the screen for $500 \mathrm{~ms}$. Then, a second face, along with a response prompt, was presented for $600 \mathrm{~ms}$. Lastly, a final fixation crosshair was presented for $300 \mathrm{~ms}$, for a total duration of $2 \mathrm{~s}$ per trial. A jittered intertrial interval (range: $0-7$, mean $=0.55, S D=1.03$ ) then followed. Participants used two fingers of their left hand to indicate, for each pair of faces, whether the second face was the same as or different from the first. Participants provided their response while the second face and response prompt were being presented on screen. The locations of both the first and second faces on the screen included a randomized horizontal offset (within a predetermined range) to minimize the interference of visual after-effects. For each participant, no single face image was used in more than one pair. The specific faces assigned to each of the conditions, as well as their pairings, were randomized between participants.

Before beginning the main task, participants practiced the task to become acquainted with trial structure and speed. In experiment 2, participants completed two rounds of practice before entering the fMRI scanner and then completed an additional practice round after entering the scanner before they started the main task. Face images used in the practice rounds were drawn from the face images that were excluded from the main task stimuli.
In experiment 2, trials were divided into two runs with an equal distribution of conditions between runs. To optimize estimation of the event-related fMRI response, conditions were intermixed in a pseudo-random order and separated by a variable, algorithm-based interstimulus interval consisting of a fixation crosshair. We used OptSeq2 (Dale, 1999) to generate sequences optimized for efficiency of the contrast (single $>$ repeated) for a first-order counterbalanced event sequence. Of these sequences, we selected four sequences that contained no more than six consecutive trials of the same race. We randomly assigned (with replacement) an event sequence for each functional run to avoid spurious results attributable to differences between conditions in one specific event sequence (Mumford et al., 2014). Within condition, trials were presented in a unique random order for each participant. During the task, we measured behavioral task performance, including accuracy and response reaction time.

\section{Face functional dynamic localizer task}

After completing the main task, participants in experiment 2 completed the dynamic localizer task (Pitcher et al., 2011) to localize brain regions associated with the processing of faces. Participants were informed about this task only on its execution. The dynamic localizer task instructed participants to respond via a button press to dynamic stimuli, short (3 s) movie clips of various categories. The stimuli were grouped into five categories: faces, objects, bodies, landscape scenes, and scrambled objects. All faces and bodies belonged to White individuals. We chose these specific stimuli as they have been previously validated in a dynamic task (Pitcher et al., 2011). To the best of our knowledge, no previous studies have validated non-White dynamic face stimuli. Each run was presented in the following structure: first, a fixation crosshair was presented for $18 \mathrm{~s}$; then, movie clips were presented back-to-back with no intertrial interval. Movie clips were blocked by category such that each block contained six video clips, of $\sim 3 \mathrm{~s}$ each, all portraying the same category (e.g., block 1 contained only clips of faces, block 2 contained only clips of objects, etc.). Then, another fixation crosshair was presented for $18 \mathrm{~s}$, followed by another series of different video clips organized like the first, but with the order of categories reversed. The run concluded with a final fixation crosshair presented for $18 \mathrm{~s}$. Each run lasted $234 \mathrm{~s}$, and there were four runs total. Participants were asked to press a button when they saw a repeated stimulus (a one-back repetition detection); for each run, there was one repetition within each category. We implemented the task by adapting code written by Visconti di Oleggio Castello (2017).

\section{Post-task behavioral measures}

Upon completion of the face task, participants in experiment 1 completed a surprise recognition memory task. For this task, two faces were presented side-by-side on a screen, with one face having been presented previously, and one face being completely novel. Thirty-six 
pairs of faces per race per gender were presented (144 face pairs in total). Participants used four keys to indicate which was the previously presented face, given four choices: surely left, maybe left, maybe right, or surely right.

Participants in both experiments also completed the following questionnaires and behavioral measures: external and internal motivation to control prejudice (Plant and Devine, 1998), social dominance orientation (Ho et al., 2015), and the implicit association test (IAT; Greenwald et al., 1998) using Black and White faces as target stimuli with positive and negative categories (Nosek et al., 2007). Finally, to assess the degree of outgroup contact, participants responded to the following three items (all requiring open-ended responses, anchored at 0): "How many African-American friends do you have?", "In a typical week, how many times do you meet with AfricanAmerican friends?", "How many of your close friends or family have African-American friends?". Aside from the recognition memory task, all postscan behavioral measures and questionnaires were included for the purposes of exploratory analysis, as noted in the preregistration. It is our intent to conduct further studies, and to eventually aggregate the results of these measures across studies once a suitable power is attained.

\section{fMRI acquisition and preprocessing}

We collected all images with a 3T Siemens Prisma scanner system (Siemens Medical Systems) using a 64-channel radiofrequency head coil. First, we acquired high-resolution anatomic images using a T1-weighted 3D MPRAGE sequence $(\mathrm{TR}=2200 \mathrm{~ms}, \mathrm{TI}=1100 \mathrm{~ms}$, acquisition matrix = $256 \times 256 \times 176$, flip angle $=7$, voxel size $=1 \times 1 \times 1 \mathrm{~mm}^{3}$ ) Second, we acquired a fieldmap in the same plane as the functional images to correct for inhomogeneities in the magnetic field (Cusack and Papadakis, 2002). Next, we collected whole brain functional images using a simultaneous multislice (multiband) T2*-weighted gradient echo sequence, sensitive to BOLD contrast, developed at the Center for Magnetic Resonance Research (CMRR) at the University of Minnesota (Feinberg et al., 2010; Moeller et al., 2010; Xu et al., 2013; TR $=2000 \mathrm{~ms}$, TE $=30 \mathrm{~ms}$, voxel size $=2 \times 2 \times 2 \mathrm{~mm}^{3}, 75$ slices auto-aligned to -25 degrees of the AC-PC line, image matrix $=104 \times 104$, $\mathrm{FOV}=208 \times 208 \mathrm{~mm}^{2}$, flip angle $=75^{\circ}$, GRAPPA acceleration factor $=2$, multiband factor $=3$, phase encoding direction $=A->P$ ). Following a short in-scanner practice scan, the face repetition suppression task included two runs consisting of 188 volumes each and was followed by the dynamic face localizer task including four runs, 120 volumes each; all runs were complemented by two additional dummy scans and an initial period of $\sim 26 \mathrm{~s}$ dedicated to references for the GRAPPA procedure. The first three volumes from each run (i.e., in addition to dummy scans) were discarded to ensure T1 equilibrium. The last five volumes from the face repetition suppression runs always included a crosshair fixation to ensure the appropriate estimation of the hemodynamic function for the last events in each run.

We conducted rudimentary quality control using the recommendations for the quality control tool implemented at the scanner facility. We used SPM12 version 6225 (Wellcome Department of Cognitive Neurology) on a 2015b MATLAB platform (MathWorks) to process and analyze the fMRI data. We corrected functional data for differences in acquisition time between slices, corrected for inhomogeneities in the magnetic field using the fieldmap (Cusack and Papadakis, 2002), realigned to the first image to correct for head movement using a second degree B-spline interpolation, unwarped to account for residual movement related variance using a fourth degree B-spline interpolation, and co-registered with each participant's anatomic data. Then, we transformed the functional data into a standard anatomic space (2-mm isotropic voxels) based on the ICBM152 brain template (Montreal Neurologic Institute). We then spatially smoothed (5- $\mathrm{mm}$ full-width at half-maximum) normalized data using a Gaussian Kernel.

\section{Statistical analysis}

\section{Statistical modeling, behavioral data}

We analyzed reaction time and accuracy data with mixed models as implemented in the Ime4 package version 1.1-14 (Bates et al., 2014) for R version 3.4.2 (R Core Team, 2017). To avoid transformation of raw reaction time data, we used generalized linear mixed models (gLMMs) with the inverse Gaussian identity link (Lo and Andrews, 2015). Memory performance in experiment 1 was analyzed using logit gLMMs with the binomial link (Jaeger, 2008). We included random effects for the intercepts for participants in all analyses. We added random intercepts for faces and by-participant random slopes for the fixed effect of race if this addition did not result in a convergence failure. Trials that elicited no response $(<1.5 \%$ of all trials; no difference between conditions) were excluded from reaction time analyses.

\section{Neuroimaging data}

We performed statistical analyses using the general linear model (GLM) that included boxcar functions of variable duration determined per trial by reaction time to target faces (i.e., variable epochs). We chose this analysis approach to control for effects of reaction time on the neural response (Grinband et al., 2008). We set the onset of the boxcar function to the onset of prime face presentation on each trial. We deviated from the preregistered protocol by modeling single-face trials as a boxcar function with a fixed duration of $600 \mathrm{~ms}$ (the duration of presentation for the first face, rather than reaction time to target face) to capture true baseline activity for a single face. The reported results replicated when we conducted the analyses without this deviation (Extended Data Fig. 3-3).

The model included six conditions per gender (two races by three conditions: repeated faces, different faces, single face). We modeled trials that elicited no response in a separate regressor, and all regressors of interest were convolved with a canonical hemodynamic response function and its temporal derivative. The final first-level GLM was high-pass filtered at $128 \mathrm{~s}$ and included nuisance regressors specifying the six motion parameters calculated during the motion correction procedures, their temporal 
derivative, and a session mean per run. Preregistered validation analyses were conducted with an additional model that included an additional separate regressor for trials in which participants erred (Extended Data Fig. 3-2).

\section{Regions of interest (ROIs)}

We defined ROls independently from the task localizer data by the group-constrained subject-specific method (Julian et al., 2012) as implemented in the spm_ss toolbox (Nieto-Castañón and Fedorenko, 2012). Briefly, this method was designed to discover regions that are systematically activated across participants and to define the borders around and between each of these regions. This method identifies key "parcels" within which most participants show activation for the contrast of interest. The selection of functional ROls for individual participants is then accomplished by intersecting each individual participant's localizer activation map with each of the parcels, thus defining functional ROls in each individual participant in a fully algorithmic fashion. We applied this method to generate ROls that responded to faces over all other categories (the face vs other contrasts; for the full results of this procedure, see Extended Data Fig. 3-6). We then extracted average parameter estimates across voxels from each participantspecific functional ROI using in-house scripts. We analyzed the data using a within-participant 2 (race) by 3 (condition) ANOVA as implemented by afex package (Singmann et al., 2018) for R, version $0.22-1$, and plotted the results using the package ggstatsplot (Patil, 2018), version 0.2.0, and the package dabestr (Ho et al., 2019), version 0.2.2.

\section{Statistical inference}

For the main analysis of interest (hypotheses 5 and 6 in the preregistration), we focused on the race by condition interaction, with specific focus on two separate interaction contrasts. One interaction contrast tested activation differences between the single-face condition and the repeatedface condition as a function of race, and another interaction contrast tested activation differences between the singleface condition and the different-face condition as a function of race. Follow-up simple effects models (one model per condition) tested differences between responses to repeated and different faces separately for Black and White faces. To demonstrate no difference between conditions, we performed an equivalence test using the equivalence package for R, version 0.7.2 (Robinson, 2016).

\section{Open practices}

Experiment 1 was not formally preregistered. All data collection procedures and analytic choices for experiment 2 were preregistered on the Open Science Framework (OSF; https://osf.io/cw4dj/). We explicitly report any deviations from preregistration in the manuscript and Extended Data. All de-identified data and code are freely available on the OSF (https://osf.io/6z5cj/).

\section{Results and Discussion}

\section{Experiment 1}

In experiment 1, we used a combination of memory and reaction time measures to validate our paradigm. Thirty-

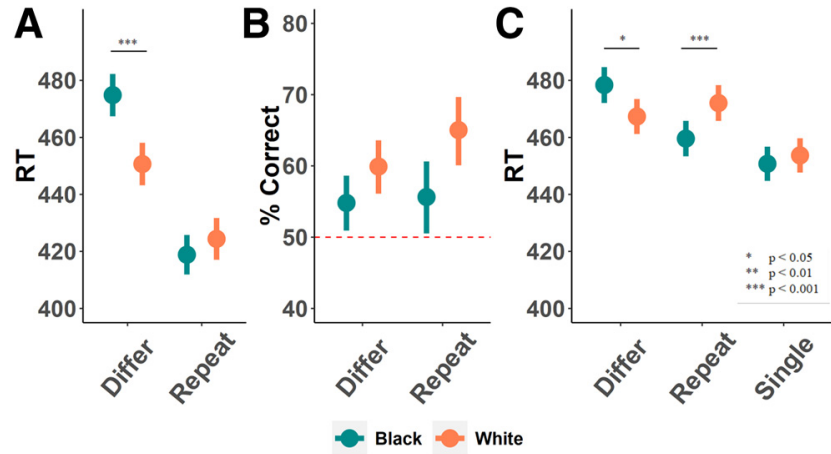

Figure 2. Reaction time and memory performance for male faces in experiments 1 and 2. All panels depict estimates computed by gLMMs, as detailed in Materials and Methods. $\boldsymbol{A}$, In the face repetition task in experiment 1 , participants responded faster to repeated faces $(t=11.33, p<0.001)$. This effect was qualified by an interaction with race, such that participants responded faster to different White individuals compared with different Black individuals (for inferential statistics, see main text). $\boldsymbol{B}$, In a two-alternative forced-choice test, participants in experiment 1 indicated their memory by selecting the individual they thought was presented in the face repetition task. All trials included one previously presented individual ("old") and one never-before-seen individual ("new"). Participants remembered old White targets better than old Black targets $(O R=1.16$, Wald's $z=3.005, p=0.003$ ). We did not observe a significant effect of type of repetition on memory $(O R=1.07$, Wald's $z=1.48$, $p=0.14)$ or of the interaction of repetition with race $(\mathrm{OR}=1.05$, Wald's $z=1.09, p=0.28$ ). $C$, Experiment 2 included an additional condition, single faces, to facilitate comparison of repetition suppression to baseline neural activity for each race. Race did not affect reaction time in the single-face condition $(t=1.04$, $p=0.300$ ). When analyzing only face-pair trials (omitting the single face trials), participants responded faster to repeated faces $(t=2.46, p=0.014)$, an effect that was qualified by an interaction, replicating the results of experiment 1 (interaction model comparison: $\left.\chi_{(1)}=15.095, p<0.001\right)$ : participants were slower to respond to different Black faces compared with different White faces $(t=2.48, p=0.013)$. Unexpectedly, participants in experiment 2 were also slower to respond to repeated White faces compared with repeated Black faces $(t=3.29, p<0.001)$. Note that unlike experiment 1 , the correct response key in experiment 2 was imbalanced between the conditions; we assigned the same key to single and different conditions to simplify the task, hence assigning the same correct response key to two thirds of the trials. This design choice probably slowed the responses to repeated trials, as participants had to use an infrequent key to respond correctly to these trials. This, in turn, might have made responses to repeated targets more difficult, a difficulty that manifested particularly strongly for White targets. Error bars indicate standard error of the mean.

eight White participants first viewed 96 repeated or different face pairs (24 per gender, per race). Subsequently, these participants completed a surprise memory task in which they saw pairs of faces, with one previously seen face and one new face in each pair, and indicated which face they had previously viewed. Because pilot testing demonstrated that participants show the effect only for male targets, we report analyses limited to these faces (for analysis of behavioral responses to female faces, see Extended Data Fig. 1-1). In line with previous studies, 
A

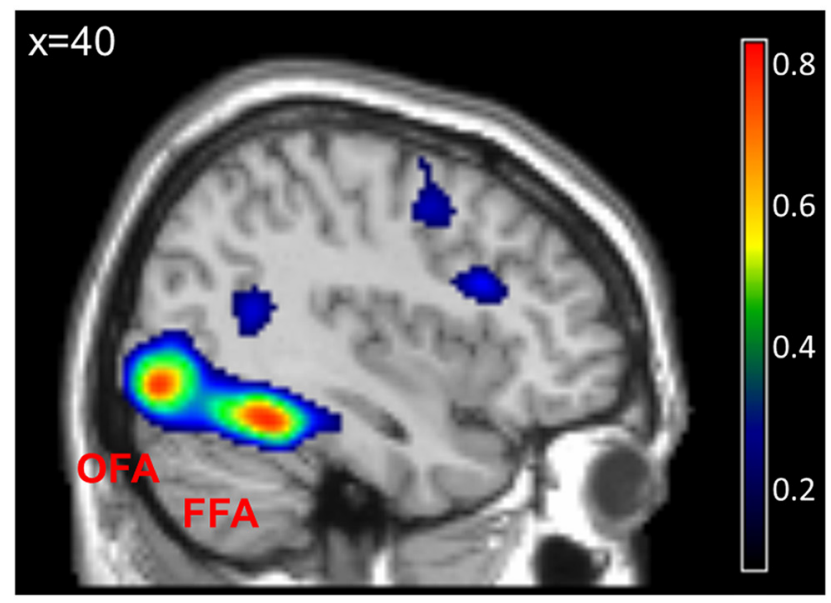

B
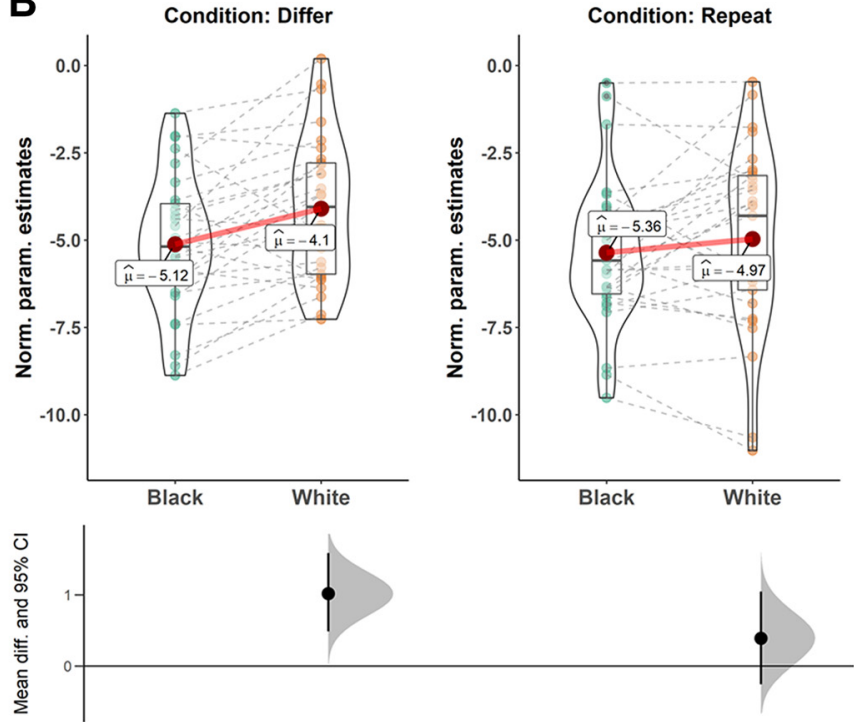

Figure 3. Results of the dynamic face localizer task and repetition suppression parameter estimates extracted from the FFA. $\boldsymbol{A}$, The spatial extent of the key ROls of interest, the FFA, and the OFA, presented as degree of spatial overlap between participants in normalized space. We generated these ROls with the group-constrained subject-specific (GcSS) approach (Julian et al., 2012) for the contrast of faces $>$ other categories at the level of each individual participant. For a table listing the full ROI list, see Extended Data Figure 3-6. B, Repetition suppression parameter estimates in the FFA for different-face and repeated-face pairs for Black and White male faces. Upper panel: to present repetition suppression effects, we subtracted neural activity in response to single faces (baseline) from the neural response to the different conditions (differ, repeat), separately for each race (Black, White). Negative values indicate neural suppression compared with baseline. Across all figures, individual dots represent neural suppression for unique participants. Each figure also visualizes the mean of each condition (as a red dot), the median (solid horizontal line), and first and third quartiles (boxplot). Lower panel: mean effect size (the difference in suppression effect) and the bootstrapped 95\% confidence intervals for the comparison between Black and White targets in each condition. The results demonstrate more release from suppression (i.e., less negative values) for different White targets compared with different Black targets, but no difference in suppression for repeating Black and White targets. This pattern suggests that the FFA forms unique representations for different individuals, but only for members of one's own racial group. We report the results of the parallel analysis in the OFA and visual cortex in Figure 4. Extended Data Figure Figures 3-1, 3-2, 3-3, 3-4 demonstrate the robustness of the effect to different analytic strategies. In Extended Data Figure 3-5, we report the results of the main analysis with female faces.

participants were more likely to accurately remember faces of White men $(61.6 \%$ correct) than faces of Black men $[55.1 \%$ correct; odds ratio $(\mathrm{OR})=1.16$, Wald's $z=3.005, p=0.003$; for full results; Fig. $2 B]$. In addition, in the face repetition task, participants were quicker to identify different White faces as different (mean \pm SE: $451 \pm$ $7 \mathrm{~ms})$ than different Black faces $(475 \pm 7 \mathrm{~ms} ; t=3.664$, $p<0.001$ ), but they identified Black and White repeated faces as identical equally fast $(419 \pm 7$ vs $424 \pm 7 \mathrm{~ms}$ for Black and White faces, respectively; $t=0.568, p>0.5$; interaction model comparison: $\chi_{(1)}=16.89, p<0.001$; Fig. $2 A)$. In other words, participants showed reduced memory and slower responses for male outgroup faces, particularly when they viewed two different individuals from each group. This suggests that although perceivers can successfully process a target from an outgroup if they see it repeatedly, they treat different outgroup faces as more homogenous to one another relative to different ingroup faces.

\section{Experiment 2}

Participants in experiment 1 demonstrated a behavioral outgroup homogeneity effect in the repetition paradigm. To examine neural representation differences between the groups, experiment 2 included 29 White participants who performed the task while undergoing fMRI scanning. We used a separate face localizer task (Pitcher et al., 2011 ) to identify a priori ROls in right fusiform gyrus (FFA) and right occipital cortex (OFA; Fig. 3A). We did not identify a robust cluster in a third hypothesized ROI, the anterior temporal lobe (for details of localization procedure, see Materials and Methods; Julian et al., 2012).

We then examined patterns of repetition suppression for repeated and different faces. For each condition of interest (repeated and different faces), we computed the differences from baseline (single face) for Black and White targets. In line with our preregistration and experiment 1 , we report the results for male targets only (for results for female targets, see Extended Data Fig. 3-5). When a second face was identical to the first, activation in FFA was suppressed to an equivalent degree for both Black and White faces [repetition effect: $F_{(1,28)}=160.20$, mean squared error $(\mathrm{MSE})=4.83, p<0.001, \eta_{\mathrm{p}}^{2}=0.85$; interaction effect: $F_{(1,28)}=1.34$, MSE $=0.82, p=0.26, \eta_{p}^{2}=0.05$; equivalence test for the two repetition effects (mean difference: 0.39): $p=0.04$, equivalence interval $(-0.96$, $0.18)]$. In other words, perceivers showed similar levels of suppression in FFA when faces were repeated, regardless of race. By contrast, FFA was released from suppression 
A

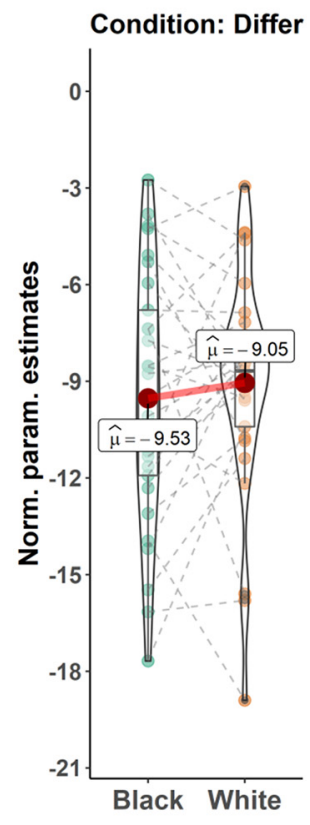

\section{OFA}

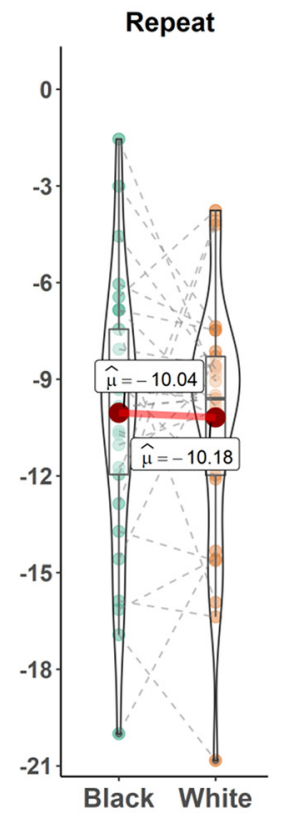

B

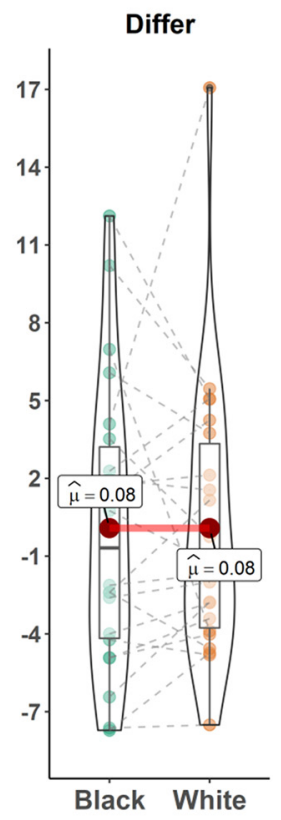

Repeat

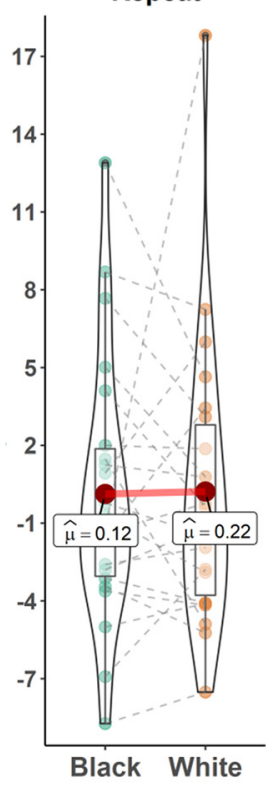

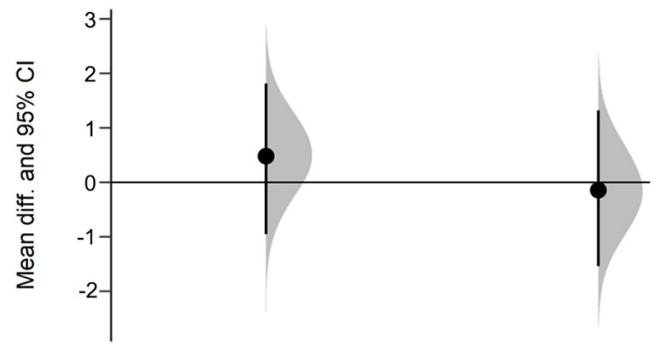

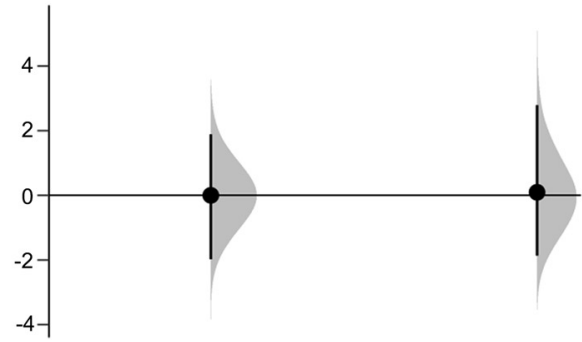

Figure 4. Repetition suppression results for repeated and different Black and White targets for the OFA and primary visual cortex (for additional details on analysis approach, see Materials and Methods). Similar to Figure 3B, negative values indicate neural suppression compared with baseline. The lower panel depicts effect size estimate (the difference in suppression effect) and the bootstrapped $95 \%$ confidence intervals for the comparison between Black and White targets in each condition. $\boldsymbol{A}$, The OFA demonstrated robust repetition suppression for repeated and different faces $\left(F_{(1,28)}=245.88, \mathrm{MSE}=12.06, p<0.001, \eta_{\mathrm{p}}^{2}=0.90\right.$ and $F_{(1,28)}=247.03$, MSE $=10.13, p<0.001, \eta_{p}^{2}=0.90$ for repeated and different targets, respectively). The OFA showed some release from suppression for different faces $\left(F_{(1,28)}=9.93\right.$, MSE $\left.=1.98, p=0.004, \eta_{\mathrm{p}}^{2}=0.26\right)$, but we found no indication for an effect of race on suppression for repeated or different targets $\left(F_{(1,28)}=0.04\right.$, MSE $=3.88, p=0.85, \eta_{\mathrm{p}}^{2}=0.001$ and $F_{(1,28)}=0.46$, MSE $=3.62, p=0.50, \eta_{\mathrm{p}}^{2}=0.02$, respectively). Thus, the OFA was not sensitive to the group features of the different faces. $\boldsymbol{B}$, Primary visual cortex (including V1) served as a control condition. No effects were observed in this region (all $F s<1.4$, all $p s>0.2$ ).

when the second face differed from the first, but only for White faces. We observed more release from suppression for White faces than for Black faces, suggesting that participants perceived White faces, but not Black faces, as representing different individuals (repetition effect: $F_{(1,28)}=13.16$, $\mathrm{MSE}=0.57, p=0.001, \eta_{\mathrm{p}}^{2}=0.32$; interaction between repeating and different faces: $F_{(1,28)}=7.64, \mathrm{MSE}=0.37$, $p=0.01, \eta_{\mathrm{p}}^{2}=0.21$; Fig. $3 B$ ). Remarkably, we observed similar levels of repetition suppression for two different Black individuals as for two identical faces [equivalence test for suppression of different and repeated Black faces (mean difference: 0.24): $p<0.001,(-0.52,0.04)$ ], which suggests that participants did not consistently perceive the two photographs to represent distinct individuals.
This pattern of results was selective for the FFA, which is thought to be the earliest visual area that encodes the unique identity of faces, rather than just their distinct perceptual features (Duchaine and Yovel, 2015). Patterns of repetition suppression did not vary for different Black and White faces in either the OFA $\left(F_{(1,28)}=0.46, \mathrm{MSE}=3.62\right.$, $\left.p=0.50, \eta_{\mathrm{p}}^{2}=0.02\right)$ or primary visual cortex $\left(F_{(1,21)}<0.01\right.$, $\mathrm{MSE}=5.28, p>0.99, \eta_{\mathrm{p}}^{2}<0.001$; Fig. 4). Finally, results were robust across several preregistered analytic variations, including analyses with excluded participants as well as when excluding trials that were answered incorrectly (see Materials and Methods; Extended Data Figs. 31, 3-2, 3-3). Together, these findings suggest that the FFA (but not lower-level visual regions) processes racial 
outgroup individuals as more similar to each other relative to ingroup individuals.

The present results expand on previous investigations in several important ways. First, most studies of the otherrace or the other-group effect have documented increased univariate activity in the FFA in response to owngroup faces compared with faces from a different social group (Golby et al., 2001; Kim et al., 2006; Van Bavel et al., 2008, 2011; Feng et al., 2011; for review, see Molenberghs and Louis, 2018). However, mean activity level can be susceptible to multiple moderators, including attention and motivation. Indeed, a recent study demonstrated that participants who experience resource scarcity demonstrate reduced FFA activity for Black faces and increased FFA activity for White faces (Krosch and Amodio, 2019). Thus, mean FFA activity can reflect the influence of contextual factors on face perception, rather than measure the representations underlying the perceived face. Here, instead of analyzing mean activity, we use the robust phenomenon of repetition suppression to demonstrate that the FFA utilizes a similar representation for different outgroup, but not ingroup, faces.

Our approach also complements a recent demonstration of differential release from suppression for Black and White targets (Hughes et al., 2019). One outstanding issue concerns the demonstration of the repetition effect. In their study, Hughes and colleagues compared blocks of repeated faces to blocks of faces morphed to different degrees, which assumes equivalent magnitude of neural activity for repeated White and Black faces without explicitly accounting for it. By contrast, our paradigm allowed us to quantify neural activity for baseline, repeated, and different face trials for Black and White faces separately, providing a straightforward index of repetition suppression and release from suppression. Second, we observed behavioral and neural outgroup homogeneity effects within the same paradigm, whereas Hughes and colleagues demonstrated neural and behavioral effects in disparate paradigms. Third, we adopted a subject-specific ROI selection approach and included exploratory analyses of female faces. Thus, our preregistered study provides a substantial extension of the existing literature by (1) conceptually replicating prior work (Hughes et al., 2019), thereby bolstering our confidence in the reliability of this effect; and (2) providing further evidence for representation-based accounts of the other-race homogeneity effect (see also Yaros et al., 2019). Notably, neither of these findings speak to the developmental origins of the effect. Specifically, individuals continuously absorb information from their environment, and their social behaviors and representations undoubtedly update as a function of this input (Rule et al., 2013). Thus, our findings cannot indicate whether the differences we observed stem from innate processes or, alternatively, were acquired throughout participants' lifetimes.

Our findings have two potential limitations. First, we observed the effects only for male and not for female targets (Fig. 3; Extended Data Fig. 3-5). One possibility is that women are less likely to be targets of the outgroup homogeneity effect. These findings accord with a broader literature documenting that outgroup men are more likely targets of intergroup discrimination and harm than are women [e.g., social dominance theory (Sidanius and Pratto, 2001) and the theory of gendered prejudice (Sidanius et al., 2019), both highlighting the importance of gender as a moderator in intergroup relations]. These findings could also indicate that representations in the FFA reflect multiple categorical geometries, including race, gender, and their interaction (Freeman et al., 2018). That said, we found that images of Black female targets in our task were more different from each other (with regard to image properties) than the other conditions. Thus, a third possibility is that increased variability made different Black female faces more distinguishable. Rather than omit female faces from the paradigm like so many other studies of this phenomenon, the current investigation included female targets to help start building a knowledge base to adjudicate among these competing explanations. A second limitation of our study is that it was constrained to White perceivers; nevertheless, given the robustness of the outgroup homogeneity effect across groups and cultures (Wan et al., 2015; Kokje et al., 2018), these results are likely to generalize to additional groups and targets (e.g., Asian perceivers viewing White faces). Testing this hypothesis remains a goal for future studies.

Many theories of human sociality begin with the assumption that perceivers can keep track of others' reputation by correctly identifying and later remembering what they did, and to whom they did it. Nevertheless, humans routinely fail to engage in such basic social cognition for outgroup members, in large part because perceivers do not consistently distinguish among individual members of such outgroups (especially those delineated by race and ethnicity). This outgroup homogeneity effect undermines one of the basic starting conditions of human (pro)sociality, in that it forestalls the ability to identify individuals with a unique set of past actions and behavioral tendencies. Here, we suggest that the potential origins of the outgroup homogeneity effect lie in failures of visual processing to form distinct representations of individual members of outgroups, something that it nevertheless accomplishes exquisitely for members of one's own social groups.

\section{References}

Bates DM, Maechler M, Bolker B, Walker S (2014) Ime4: linear mixed-effects models using Eigen and S4. Available at http://cran. r-project.org/package $=$ Ime 4 .

Behrman BW, Davey SL (2001) Eyewitness identification in actual criminal cases: an archival analysis. Law Hum Behav 25:475-491.

Boldry JG, Gaertner L, Quinn J (2007) Measuring the measures: a meta-analytic investigation of the measures of outgroup homogeneity. Gr Process Intergr Relations 10:157-178.

Cohen J (1988) Statistical power analysis for the behavioral sciences, Ed 2. Mahwah: Lawrence Erlbaum Associates, Inc.

Correll J, Hudson SM, Guillermo S, Earls HA (2017) Of kith and kin: perceptual enrichment, expectancy, and reciprocity in face perception. Pers Soc Psychol Rev 21:336-360.

Cusack R, Papadakis N (2002) New robust 3-D phase unwrapping algorithms: application to magnetic field mapping and undistorting echoplanar images. Neuroimage 16:754-764.

Dale AM (1999) Optimal experimental design for event-related fMRI. Hum Brain Mapp 8:109-114. 
Duchaine B, Yovel G (2015) A revised neural framework for face processing. Annu Rev Vis Sci 1:393-416.

Feinberg DA, Moeller S, Smith SM, Auerbach E, Ramanna S, Gunther M, Glasser MF, Miller KL, Ugurbil K, Yacoub E (2010) Multiplexed echo planar imaging for sub-second whole brain FMRI and fast diffusion imaging. PLoS One 5:e15710.

Feng L, Liu J, Wang Z, Li J, Li L, Ge L, Tian J, Lee K (2011) The other face of the other-race effect: an fMRI investigation of the otherrace face categorization advantage. Neuropsychologia 49:37393749.

Freeman JB, Stolier RM, Brooks JA, Stillerman BS (2018) The neural representational geometry of social perception. Curr Opin Psychol 24:83-91.

Gilaie-Dotan S, Gelbard-Sagiv H, Malach R (2010) Perceptual shape sensitivity to upright and inverted faces is reflected in neuronal adaptation. Neuroimage 50:383-395.

Golby AJ, Gabrieli JDE, Chiao JY, Eberhardt JL (2001) Differential responses in the fusiform region to same-race and other-race faces. Nat Neurosci 4:845-850.

Gotts SJ, Chow CC, Martin A (2012) Repetition priming and repetition suppression: a case for enhanced efficiency through neural synchronization. Cogn Neurosci 3:227-237.

Greenwald AG, Mcghee DE, Schwartz JLK (1998) Measuring individual differences in implicit cognition: the implicit association test. J Pers Soc Psychol 74:1464-1480.

Grill-Spector K, Kushnir T, Edelman S, Avidan G, Itzchak Y, Malach R (1999) Differential processing of objects under various viewing conditions in the human lateral occipital complex. Neuron 24:187203.

Grinband J, Wager TD, Lindquist M, Ferrera VP, Hirsch J (2008) Detection of time-varying signals in event-related $\mathrm{fMRI}$ designs. Neuroimage 43:509-520.

Ho AK, Sidanius J, Kteily N, Sheehy-Skeffington J, Pratto F, Henkel KE, Foels R, Stewart AL (2015) The nature of social dominance orientation: theorizing and measuring preferences for intergroup inequality using the new $\mathrm{SDO}_{7}$ scale. J Pers Soc Psychol 109:10031028.

Ho J, Tumkaya T, Aryal S, Choi H, Claridge-Chang A (2019) Moving beyond $P$ values: data analysis with estimation graphics. Nat Methods 16:565-566.

Hugenberg K, Wilson JP, See PE, Young SG (2013) Towards a synthetic model of own group biases in face memory. Vis Cogn 21:1392-1417.

Hughes BL, Camp NP, Gomez J, Natu VS, Grill-spector K, Eberhardt $\mathrm{JL}$ (2019) Neural adaptation to faces reveals racial outgroup homogeneity effects in early perception. Proc Natl Acad Sci USA 116:14532-14537.

Jaeger TF (2008) Categorical data analysis: away from ANOVAs (transformation or not) and towards logit mixed models. J Mem Lang 59:434-446.

Judd CM, Park B, Yzerbyt V, Gordijn EH, Muller D (2005) Attributions of intergroup bias and outgroup homogeneity to ingroup and outgroup others. Eur J Soc Psychol 35:677-704.

Julian JB, Fedorenko E, Webster J, Kanwisher N (2012) An algorithmic method for functionally defining regions of interest in the ventral visual pathway. Neuroimage 60:2357-2364.

Kim JS, Yoon HW, Kim BS, Jeun SS, Jung SL, Choe BY (2006) Racial distinction of the unknown facial identity recognition mechanism by event-related fMRI. Neurosci Lett 397:279-284.

Kokje E, Bindemann M, Megreya AM (2018) Cross-race correlations in the abilities to match unfamiliar faces. Acta Psychol (Amst) 185:13-21.

Krosch AR, Amodio DM (2019) Scarcity disrupts the neural encoding of Black faces: a socioperceptual pathway to discrimination. $J$ Pers Soc Psychol 117:859-875.

Lo S, Andrews S (2015) To transform or not to transform: using generalized linear mixed models to analyse reaction time data. Front Psychol 6:1-16.
Ma DS, Correll J, Wittenbrink B (2015) The Chicago face database: a free stimulus set of faces and norming data. Behav Res Methods 47:1122-1135.

Malpass RS, Kravitz J (1969) Recognition for faces of own and other race. J Pers Soc Psychol 13:330-334.

Marcon JL, Meissner CA, Frueh M, Susa KJ, MacLin OH (2010) Perceptual identification and the cross-race effect. Vis Cogn 18:767-779.

Mathur VA, Harada T, Chiao JY (2012) Racial identification modulates default network activity for same and other races. Hum Brain Mapp 33:1883-1893.

Meissner CA, Brigham JC (2001) Thirty years of investigating the own-race bias in memory for faces: a meta-analytic review. Psychol Public Policy Law 7:3-35.

Moeller S, Yacoub E, Olman CA, Auerbach E, Strupp J, Harel N, Uğurbil K (2010) Multiband multislice GE-EPI at 7 tesla, with 16fold acceleration using partial parallel imaging with application to high spatial and temporal whole-brain fMRI. Magn Reson Med 63:1144-1153.

Molenberghs P, Louis WR (2018) Insights from fMRI studies into ingroup bias. Front Psychol 9:1-12.

Mumford JA, Davis T, Poldrack RA (2014) The impact of study design on pattern estimation for single-trial multivariate pattern analysis. Neuroimage 103:130-138.

Nieto-Castañón A, Fedorenko E (2012) Subject-specific functional localizers increase sensitivity and functional resolution of multisubject analyses. Neuroimage 63:1646-1669.

Nosek BA, Smyth FL, Hansen JJ, Devos T, Lindner NM, Ranganath KA, Smith CT, Olson KR, Chugh D, Greenwald AG, Banaji MR (2007) Pervasiveness and correlates of implicit attitudes and stereotypes. Eur Rev Soc Psychol 18:36-88.

Papesh MH, Goldinger SD (2010) A multidimensional scaling analysis of own- and cross-race face spaces. Cognition 116:283-288.

Park B, Rothbart M (1982) Perception of out-group homogeneity and levels of social categorization: memory for the subordinate attributes of in-group and out-group members. J Pers Soc Psychol 42:1051-1068.

Patil I (2018) ggstatsplot: "ggplot2" based plots with statistical details. Available at https://cran.r-project.org/package=ggstatsplot.

Peirce JW (2007) PsychoPy-Psychophysics software in Python. J Neurosci Methods 162:8-13.

Pitcher D, Dilks DD, Saxe RR, Triantafyllou C, Kanwisher N (2011) Differential selectivity for dynamic versus static information in face-selective cortical regions. Neuroimage 56:2356-2363.

Plant EA, Devine PG (1998) Internal and external motivation to respond without prejudice. J Pers Soc Psychol 75:811-832.

Pourtois G, Schwartz S, Seghier ML, Lazeyras F, Vuilleumier P (2005) View-independent coding of face identity in frontal and temporal cortices is modulated by familiarity: an event-related fMRI study. Neuroimage 24:1214-1224.

R Core Team (2017) R: A language and environment for statistical computing. R Foundation for Statistical Computing, Vienna, Austria. Available at https://www.R-project.org/.

Robinson A (2016) Package "equivalence." Available at https://cran. $r$-project.org/package=equivalence.

Rule NO, Freeman JB, Ambady N (2013) Culture in social neuroscience: a review. Soc Neurosci 8:3-10.

Sidanius J, Pratto F (2001) Social dominance: an intergroup theory of social hierarchy and oppression. Cambridge: Cambridge University Press.

Sidanius J, Hudson S-K, Davis G, Bergh R (2019) The theory of gendered prejudice: a social dominance and intersectionalist perspective. In: The Oxford handbook of behavioral political science (Mintz A, Terris L, eds). Oxford: Oxford University Press.

Singmann H, Bolker B, Westfall J, Aust F (2018) afex: analysis of factorial experiments. Available at https://cran.r-project.org/package= afex.

Van Bavel JJ, Packer DJ, Cunningham WA (2008) The neural substrates of in-group bias. Psychol Sci 19:1131-1139. 
Van Bavel JJ, Packer DJ, Cunningham WA (2011) Modulation of the fusiform face area following minimal exposure to motivationally relevant faces: evidence of in-group enhancement (not out-group disregard). J Cogn Neurosci 23:3343-3354.

Visconti di Oleggio Castello M (2017) Dynamic face/body/scene localizer. Available at https://github.com/mvdoc/pitcher_localizer.

Vizioli L, Rousselet GA, Caldara R (2010) Neural repetition suppression to identity is abolished by other-race faces. Proc Natl Acad Sci USA 107:20081-20086.

Wagner JK, Yu JH, Ifekwunigwe JO, Harrell TM, Bamshad MJ, Royal CD (2017) Anthropologists' views on race, ancestry, and genetics. Am J Phys Anthropol 162:318-327.

Wan L, Crookes K, Reynolds KJ, Irons JL, Mckone E (2015) A cultural setting where the other-race effect on face recognition has no social-motivational component and derives entirely from lifetime perceptual experience. Cognition 144:91-115.

Wang Z, Bovik AC, Sheikh HR, Simoncelli EP (2004) Image quality assessment: from error visibility to structural similarity. IEEE Trans Image Process 13:600-612.

Willenbockel V, Sadr J, Fiset D, Horne GO, Gosselin F, Tanaka JW (2010) Controlling low-level image properties: the SHINE toolbox. Behav Res Methods 42:671-684.

Xu J, Moeller S, Auerbach EJ, Strupp J, Smith SM, Feinberg DA, Yacoub E, Uğurbil K (2013) Evaluation of slice accelerations using multiband echo planar imaging at 3 T. Neuroimage 83:9911001.

Yaros JL, Salama DA, Delisle D, Larson MS, Miranda BA, Yassa MA (2019) A memory computational basis for the other-race effect. Sci Rep 9:19399. 